\begin{tabular}{|c|l|}
\hline Title & $\begin{array}{l}\text { Mechanism and Experimental Observability of Global Switching Between Reactive and Nonreactive Coordinates at } \\
\text { High Total Energies }\end{array}$ \\
\hline Author(s) & Teramoto, Hiroshi; Toda, Mikito; Takahashi, Masahiko; Kono, Hirohiko; Komatsuzaki, Tamiki \\
\hline Citation & $\begin{array}{l}\text { Physical Review Letters, 115(9), 093003-1-093003-5 } \\
\text { https://doi.org/10.1103/PhysRevLett.115.093003 }\end{array}$ \\
\hline Issue Date & 2015-08-28 \\
\hline Doc URL & http://hdl.handle.net/2115/63191 \\
\hline Rights & ○ 2015 A merican Physical Society \\
\hline Type & article \\
\hline File Information & PhysRevLett.115.093003.pdf \\
\hline
\end{tabular}

Instructions for use 


\title{
Mechanism and Experimental Observability of Global Switching Between Reactive and Nonreactive Coordinates at High Total Energies
}

\author{
Hiroshi Teramoto, ${ }^{1, *}$ Mikito Toda, ${ }^{2}$ Masahiko Takahashi, ${ }^{3}$ Hirohiko Kono, ${ }^{4}$ and Tamiki Komatsuzaki ${ }^{1}$ \\ ${ }^{1}$ Molecule \& Life Nonlinear Sciences Laboratory, Research Institute for Electronic Science, \\ Hokkaido University, Kita 20 Nishi 10, Kita-ku, Sapporo 001-0020, Japan \\ ${ }^{2}$ Nonequilibrium Dynamics Laboratory, Faculty Division of Natural Sciences, Research Group of Physics, \\ Nara Women's University, Nara 630-8506, Japan \\ ${ }^{3}$ Institute of Multidisciplinary Research for Advanced Materials, Tohoku University, Sendai 980-8577, Japan \\ ${ }^{4}$ Department of Chemistry, Graduate School of Science, Tohoku University, Sendai 980-8578, Japan
}

(Received 2 June 2015; published 28 August 2015)

\begin{abstract}
We present a mechanism of global reaction coordinate switching, namely, a phenomenon in which the reaction coordinate dynamically switches to another coordinate as the total energy of the system increases. The mechanism is based on global changes in the underlying phase space geometry caused by a switching of dominant unstable modes from the original reactive mode to another nonreactive mode in systems with more than 2 degrees of freedom. We demonstrate an experimental observability to detect a reaction coordinate switching in an ionization reaction of a hydrogen atom in crossed electric and magnetic fields. For this reaction, the reaction coordinate is a coordinate along which electrons escape and its switching changes the escaping direction from the direction of the electric field to that of the magnetic field and, thus, the switching can be detected experimentally by measuring the angle-resolved momentum distribution of escaping electrons.
\end{abstract}

DOI: 10.1103/PhysRevLett.115.093003

PACS numbers: 32.80.Rm, 05.45.Ac, 45.20.Jj, 82.20.-w

In the conventional picture of chemical reactions, a system starts from a reactant and ends up with a product by overcoming a first-rank saddle in between the two. If the total energy of a system is close enough to the potential energy of the saddle, a harmonic approximation of the potential energy surface at the saddle is valid during the time interval in which the system traverses through the saddle. Then, the direction toward which the reaction occurs is determined by the unstable mode at the saddle. As the total energy increases, the nonlinearity of the potential energy starts to play an important role even in the vicinity of the saddle, and the nonlinear couplings between the unstable mode and the other modes become no longer negligible.

At the total energy in which the harmonic terms dominate the nonlinear terms, one can still take them into account in a perturbative manner [1-5] so that the resulting perturbed unstable mode is decoupled from the other perturbed modes and the perturbed unstable mode is hyperbolic. A key geometrical object behind the perturbed modes is a normally hyperbolic invariant manifold (NHIM) $[6,7]$, defined as a zero level set of the perturbed unstable mode and its conjugate momentum on an equienergy surface [5]. The NHIM has a pair of stable and unstable normal directions that are linear combinations of this perturbed unstable mode and its conjugate momentum. There, the stable and unstable manifolds emanating in the normal directions determine the directions toward which the reaction occurs. Specifically, if the NHIM has a topology of a hypersphere, these manifolds are cylindrical and, thus, are called a cylindrical stable manifold and a cylindrical unstable manifold, respectively. In that case, every reactive trajectory should run through the cylindrical stable manifold and then run through the cylindrical unstable manifold [5,8-12].

If the total energy increases even further, the perturbative construction of the NHIM starts to fail even with a Padé approximation [13] and the underlying geometrical object, the NHIM, also breaks down [14,15]. The NHIM is persistent as long as its normal stability and instability dominates its tangential stability and instability, respectively. However, as the total energy increases, its tangential stability and instability may become dominant due to the nonlinear resonance within modes other than the unstable mode.

One of the physical manifestations of the breakdown of the NHIM is reaction coordinate switching [14,15] in which the reaction coordinate along which a reaction proceeds dynamically switches to a nonreactive coordinate as the total energy of the system increases, involving a change in the associating reactant, product, and transition state in between the two. This phenomenon is in opposition to the conventional picture of chemical reactions, i.e., the concept that the reactant, product, and transition state is solely determined by the topography of the potential energy surface and, thus, opens a new way to control the chemical reaction in terms of the underlying phase space geometry. The main subject of this Letter is to present a mechanism of 
reaction coordinate switching and to discuss its experimental observability.

A hydrogen atom in crossed electric and magnetic fields is one of the simplest atomic systems and, thus, has been used as an ideal benchmark for validating theoretical predictions [16-18]. In addition, in this system, by choosing the magnitudes of the electric and magnetic fields properly, an effective Planck constant can be made arbitrarily small so that the dynamics is approximated well by the classical mechanics. For this reason, this system is one of the most ideal systems for the main subject.

The Hamiltonian for a hydrogen atom in crossed electric and magnetic fields is expressed by

$H=\frac{1}{2}\left(P_{1}-\frac{\omega_{\mathrm{c}} x_{2}}{2}\right)^{2}+\frac{1}{2}\left(P_{2}+\frac{\omega_{\mathrm{c}} x_{1}}{2}\right)^{2}+\frac{1}{2} P_{3}^{2}-\frac{1}{R}-\epsilon x_{1}$,

in the atomic unit system, where $x_{i}, P_{i}(i=1,2,3)$ are the $i$ th Cartesian coordinate and its conjugate momentum, $R=\sqrt{\left(x_{1}-x_{s}\right)^{2}+x_{2}^{2}+x_{3}^{2}}, x_{s}=-\epsilon^{1 / 2}$, and $\omega_{\mathrm{c}}$ is the cyclotron frequency. This system has a saddle point at the origin which is called the Stark saddle that connects two distinct states: one in which the electron is bound to the nuclei and the other in which it is not. This saddle is of rank 1 ; that is, only one mode is unstable leaving to either of the states, while all of the other modes are stable. By using a scaling transformation,

$$
\begin{aligned}
x_{i}{ }^{\prime} & =\omega_{\mathrm{c}}^{2 / 3} x_{i}, & P_{i}{ }^{\prime} & =\omega_{\mathrm{c}}^{-1 / 3} P_{i}, \\
H^{\prime} & =\omega_{\mathrm{c}}^{-2 / 3} H, & \epsilon^{\prime} & =\omega_{\mathrm{c}}^{-4 / 3} \epsilon,
\end{aligned}
$$

the resulting Hamiltonian is

$$
H^{\prime}=\frac{1}{2}\left(P_{1}^{\prime}-\frac{x_{2}^{\prime}}{2}\right)^{2}+\frac{1}{2}\left(P_{2}^{\prime}+\frac{x_{1}^{\prime}}{2}\right)^{2}+\frac{1}{2} P_{3}^{2}-\frac{1}{R^{\prime}}-\epsilon^{\prime} x_{1}{ }^{\prime},
$$

where the (scaled) electric field $\epsilon^{\prime}$ is the only remaining parameter [5]. In what follows, we omit ' for simplicity. Followed by a coordinate transformation, the quadratic part of the Hamiltonian around the saddle can be diagonalized by a linear transformation [5]. The resulting Hamiltonian can be expressed by

$H(\xi, \eta, \mathbf{p}, \mathbf{q})=\lambda \xi \eta+\sum_{\ell=1}^{2} \frac{\omega_{\ell}}{2}\left(p_{\ell}^{2}+q_{\ell}^{2}\right)+\sum_{i, j \geq 0} c_{i j}(\mathbf{p}, \mathbf{q}) \xi^{i} \eta^{j}$,

where $\xi$ and $\eta$ denote the linear combinations of the normal coordinate and conjugate momentum of the reactive mode defined at the saddle, and $p_{\ell}$ and $q_{\ell}$ are the normal coordinates and the conjugate momenta of the nonreactive modes, respectively. In addition, the terms $c_{i j}(\mathbf{p}, \mathbf{q}) \xi^{i} \eta^{j}$ start from the order of 3 with respect to $(\xi, \eta, \mathbf{p}, \mathbf{q})$. $\lambda$ is a curvature of the potential energy surface along the unstable normal coordinate, and $\omega_{1}$ and $\omega_{2}$ are two frequencies of the nonreactive normal coordinates, which can be adjusted by $\epsilon$ [5]. Here, we chose $\epsilon$ so that $\omega_{1}$ and $\omega_{2}$ satisfy $2: 1$ resonance, i.e., $\omega_{2} / \omega_{1}=1 / 2$, in order that the two nonreactive modes can exchange energies through the resonance. If the total energy of the system, $E$, is sufficiently close to that of the saddle point, $(\xi, \eta)$ can be regarded as the reaction mode.

In the saddle region, the NHIM can be extracted by transforming Eq. (4) to the following form [19]:

$$
\bar{H}(\bar{\xi}, \bar{\eta}, \overline{\mathbf{p}}, \overline{\mathbf{q}})=\lambda \bar{\xi} \bar{\eta}+\sum_{\ell=1}^{2} \frac{\omega_{\ell}}{2}\left(\bar{p}_{\ell}^{2}+\bar{q}_{\ell}^{2}\right)+\sum_{\substack{i+j \geq 2 \\ i, j \geq 0}} \bar{c}_{i j}(\overline{\mathbf{p}}, \overline{\mathbf{q}}) \bar{\xi}^{i} \bar{\eta}^{j} .
$$

Here, again, the order of $\bar{c}_{i j}(\overline{\mathbf{p}}, \overline{\mathbf{q}}) \bar{\xi}^{i} \bar{\eta}^{j}$ is not less than 3 with respect to $(\bar{\xi}, \bar{\eta}, \overline{\mathbf{p}}, \overline{\mathbf{q}})$. This Hamiltonian, Eq. (5), satisfies

$$
\left.\frac{\partial \bar{H}(\bar{\xi}, \bar{\eta}, \overline{\mathbf{p}}, \overline{\mathbf{q}})}{\partial \bar{\xi}}\right|_{\bar{\xi}=\bar{\eta}=0}=\left.\frac{\partial \bar{H}(\bar{\xi}, \bar{\eta}, \overline{\mathbf{p}}, \overline{\mathbf{q}})}{\partial \bar{\eta}}\right|_{\bar{\xi}=\bar{\eta}=0}=0
$$

for all $\overline{\mathbf{p}}$ and $\overline{\mathbf{q}}$ and, thus, a subset of phase space that satisfies $\bar{\xi}=\bar{\eta}=0$ is an invariant set of the system. The NHIM is defined by the intersection between an equienergy surface and the invariant subset.

In Fig. 1(a), we show cylindrical stable and unstable manifolds [8-10] emanating from the NHIM, projected on the coordinate space, at the total energy $E=0.001$ above the Stark saddle. The proton is indicated by the yellow circle and the NHIM by the blue dot. The cylindrical stable manifold leading to the NHIM starting from the bound state is indicated by red and the cylindrical unstable manifold leading to the dissociation state by green. Every escaping electron should run through the red cylinder and should then run through the green cylinder to escape from the proton. Note that, around this energy, these two cylinders are emanating toward the $\xi$ direction, which is in the $x_{1}-x_{2}$ plane. This is because if the total energy is sufficiently close to that of the Stark saddle, the first and second terms in Eq. (4) provide a good approximation of the Hamiltonian in the vicinity of the Stark saddle.

However, when the total energy is far above the saddle $(E=0.71)$, the NHIM breaks down $[14,15]$. Moreover, the intersection between the equienergy surface and the invariant plane $x_{3}=P_{3}=0$ (which is invariant due to the symmetry of the system) instead becomes another NHIM in a neighborhood of the unstable periodic orbit located in the intersection between the original NHIM and the invariant plane at the total energy $E=0.99[14,15]$. The invariant plane becomes normally hyperbolic in a much broader phase space region than the neighborhood of the unstable periodic orbit, which will be shown later. In the region, its normal stretching and contracting rates dominate its tangential ones. There, its stable and unstable manifolds 
(a)

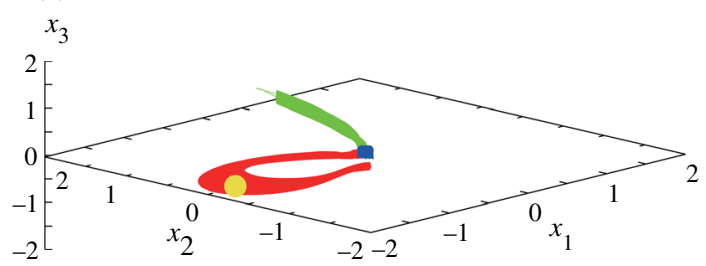

(b)

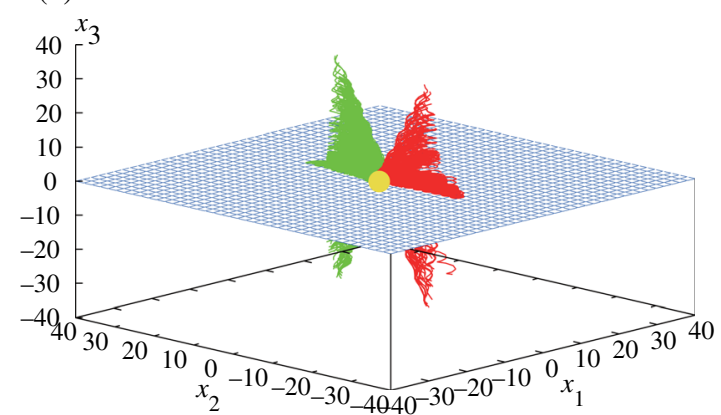

FIG. 1 (color online). (a) The cylindrical stable and unstable manifolds (indicated by red and green, respectively) emanating from the NHIM (indicated by the blue dot), projected on the coordinate space, at the total energy $E=0.001$ above the Stark saddle. The proton is indicated by the yellow circle. (b) The stable and unstable manifolds (indicated by red and green, respectively) of the invariant plane (indicated by blue) at the total energy $E=1.45$, projected on the coordinate space.

emanating from normal directions to the invariant plane determine directions toward which the reaction occurs. In Fig. 1(b), these stable and unstable manifolds at the energy $E=1.45$, projected on the coordinate space, are shown by red and green, respectively. These stable and unstable manifolds are constructed as follows: linear approximations of local stable and unstable manifolds of the invariant plane are constructed on the invariant plane by using Oseledec decomposition $[20,21]$. Then, the local stable and unstable manifolds are propagated backward and forward in time to extend them to the global stable and unstable manifolds. These two manifolds are no longer cylindrical, but trajectories in the vicinity of these manifolds are expected to follow these manifold due to the $\lambda$ lemma [22]. Figure 1(b) shows that these stable and unstable manifolds emanate toward the $x_{3}$ direction, which is perpendicular to the original $\xi$ direction. Because of this change, the directions that an electron approaches or escapes from the proton switch from the $\xi$ direction to the $x_{3}$ direction.

In Figs. 2(a) and 2(b), we show the ratio between the normal and tangential stretching rates of the invariant plane on the Poincaré surface, $x_{2}=0,\left(d x_{2} / d t\right)>0$, at the total energies $E=0.5$ and $E=1.45$. The normal (tangential) stretching rate is the longtime asymptotic growth rate of a distance between two adjacent trajectories whose initial conditions are separated infinitesimally in the normal (tangential) direction to the invariant plane. In Figs. 2(a) and 2(b), the unstable periodic orbit is indicated by the green cross and the region where the invariant plane is normally hyperbolic; i.e., the normal stretching rate that dominates the tangential stretching rate is indicated by red, whereas that in the reverse case is indicated by blue. Figure 2(b) shows that the invariant plane becomes normally hyperbolic over a broader region than that in Fig. 2(a). To evaluate it more quantitatively, in Fig. 2(c), we show a volume fraction of the normally hyperbolic region in the invariant plane. Figure 2(c) indicates that the volume fraction at the energy $E=0.5$ is only $3 \%$, whereas that at the energy $E=1.45$ is almost $50 \%$ and the onset of
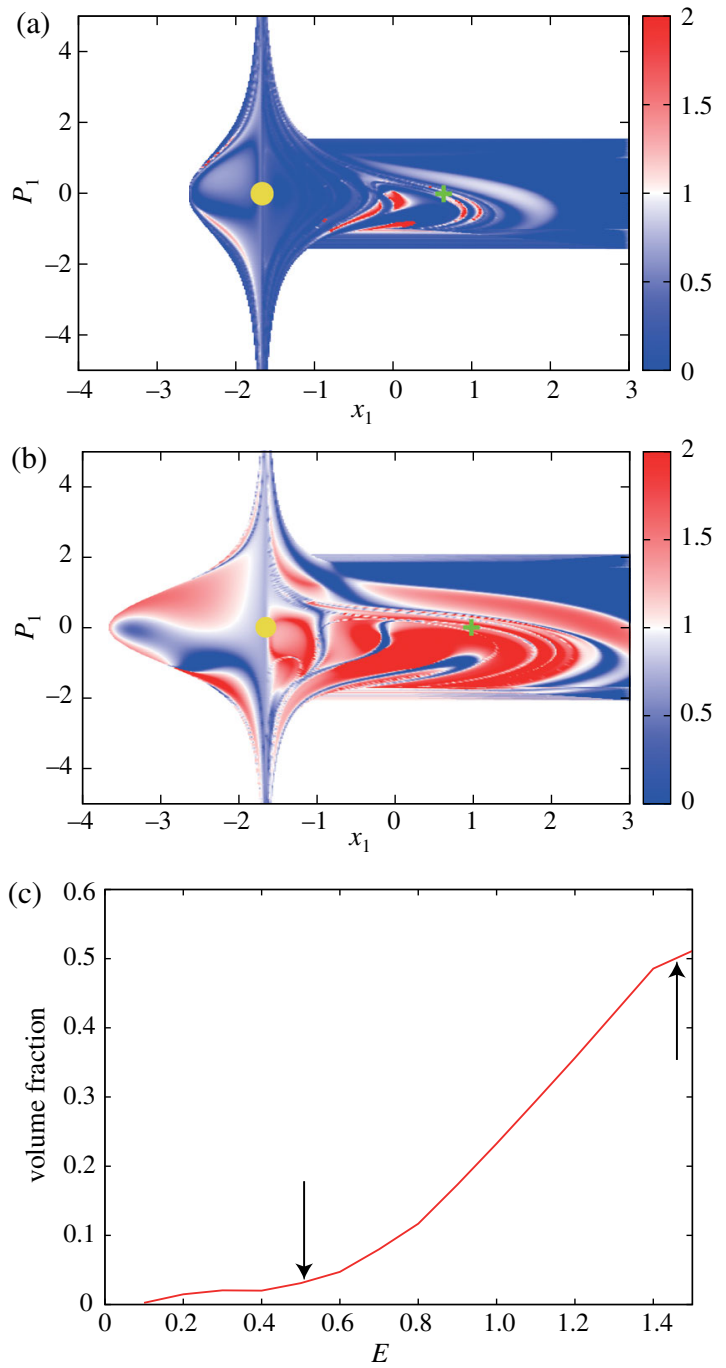

FIG. 2 (color online). (a),(b) Ratio between the normal and tangential stretching rates of the invariant plane on the Poincaré surface $x_{2}=0,\left(d x_{2} / d t\right)>0$, at the total energy (a) $E=0.5$ and (b) $E=1.45$. The proton is indicated by the yellow circle and the periodic orbit by the green cross in each figure. (c) Volume fraction in the invariant plane where the normal stretching rate dominates the tangential stretching rate. Those at energies $E=0.5$ and $E=1.45$ are indicated by the arrows. The standard error of the volume fraction is less than $3.16 \times 10^{-3}$ for all of the energies. 
the increase is at around $E=0.6$. This result indicates that the invariant plane becomes normally hyperbolic in a much broader phase space region than the neighborhood of the unstable periodic orbit, which is a mechanism of the reaction coordinate switching.

To investigate experimental observability of the reaction coordinate switching, we show in Fig. 3(a) distributions of the $P_{3}$ values of the escaping electrons at the total energies $E=0.3,0.5,0.7,1.0,1.2,1.5$, starting in the vicinity of the proton. The distribution is calculated by using 300000 randomly sampled trajectories and a bin size of 0.03 for each energy. In this system, if the distance between the electron and the proton, $R$, is sufficiently large, $P_{3}$ is nearly a constant of motion and, thus, its asymptotic value is well defined. In this calculation, we define the asymptotic region as the region where the Coulombic potential energy is less than $0.01 \%$ of the total energy. If the NHIM exists, the electron escapes toward the $\xi$ direction, which is in the $x_{1}-x_{2}$ plane, and, thus, the value of $P_{3}$ is expected to be distributed around $P_{3} \approx 0$. Instead, at the energy above the switching, the electron escapes toward the $x_{3}$ direction and, thus, the distribution is expected to have shoulders at the finite values of $P_{3}$. Figure 3 indicates that this is the case
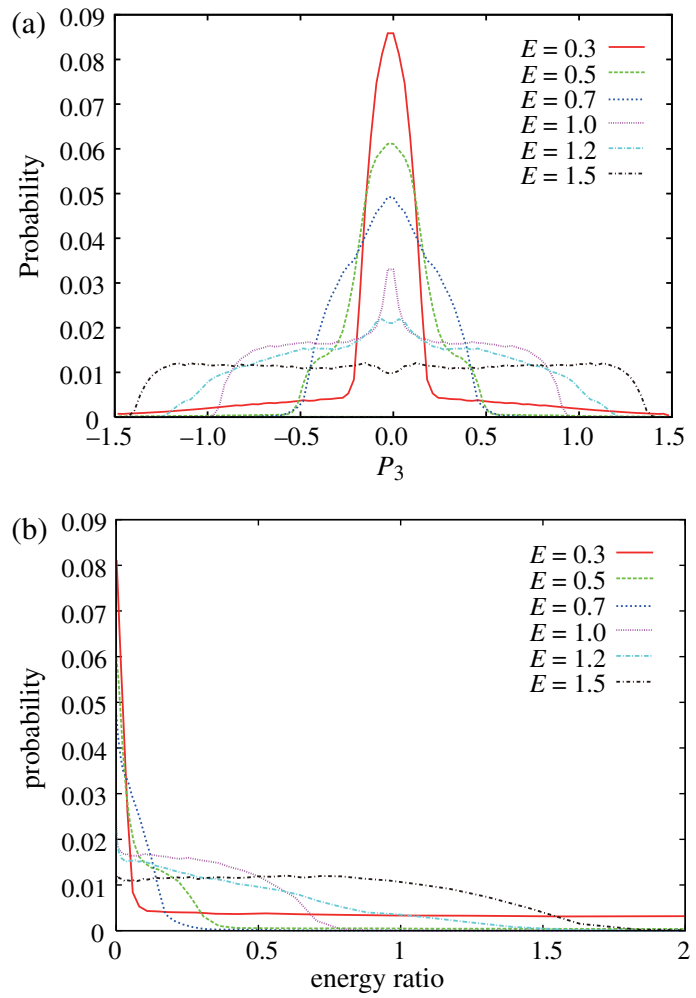

FIG. 3 (color online). (a) Distributions of the $P_{3}$ values of the escaping electrons at the total energies $E=0.3,0.5,0.7,1.0,1.2$, 1.5. (b) Distributions of the energy ratio between the energy of the $x_{3}$ component, $E_{3}$, and that of the others, $E_{12}$, of the escaping electrons at the total energies $E=0.3,0.5,0.7,1.0,1.2,1.5$. In these figures, the standard error is less than $1.73 \times 10^{-4}$ for all of the $P_{3}$ values and the energies. and the distribution starts to have shoulders in between the total energies $E=0.7$ and $E=1.0$. The energy $E=0.7$ is close to the energy $E=0.71$ at which the NHIM breaks down along several homoclinic orbits [15] and this suggests that the emergence of the shoulders is triggered by the breakdown of the NHIM. To detect the shoulders experimentally by using an angular resolved detector, not only the magnitude of $P_{3}$ but also the ratio of $P_{3}$ to the other components, $P_{1}$ and $P_{2}$, needs to be large enough. In the current setting, $P_{1}$ and $P_{2}$ are not constants of motion even in the asymptotic region and they do not have well-defined asymptotic values. Instead, we show the histogram against the energy ratio between the energy of the $x_{3}$ component, $E_{3}=\frac{1}{2} P_{3}^{2}$, and that of the others, $E_{12}=\frac{1}{2}\left[P_{1}-\left(x_{2} / 2\right)\right]^{2}+\frac{1}{2}\left[P_{2}+\left(x_{1} / 2\right)\right]^{2}-\epsilon x_{1}$, of the escaping electron in the asymptotic region in Fig. 3(b). Both of them are approximately constants of motion in the asymptotic region and thus have well-defined asymptotic values. Figure 3(b) indicates that the energy ratios are sharply distributed around 0.0 for $E=0.3$ and 0.5 (the half widths at half maximum of their distribution are less than 0.1 ), while those of the others have half widths at half maximum larger than 0.6.

In this analysis, we did not take quantum effects into account. To investigate this, we derive a scaled quantum Hamiltonian corresponding to Eq. (3). By applying a scaling transformation to the quantum Hamiltonian corresponding to Eq. (2),

$$
x_{i}^{\prime}=\omega_{\mathrm{c}}^{2 / 3} x_{i}, \quad \hat{H}^{\prime}=\omega_{\mathrm{c}}^{-2 / 3} \hat{H}, \quad \epsilon^{\prime}=\omega_{\mathrm{c}}^{-4 / 3} \epsilon,
$$

the resulting Hamiltonian becomes

$$
\begin{aligned}
\hat{H}^{\prime}= & -\frac{\hbar_{\mathrm{eff}}^{2}}{2}\left(\frac{\partial^{2}}{\partial x_{1}^{\prime 2}}+\frac{\partial^{2}}{\partial x_{2}^{\prime 2}}+\frac{\partial^{2}}{\partial x_{3}^{\prime 2}}\right)-\frac{1}{R^{\prime}} \\
& +\frac{\hbar_{\mathrm{eff}}}{2 i}\left(x_{1}^{\prime} \frac{\partial}{\partial x_{2}^{\prime}}-x_{2}^{\prime} \frac{\partial}{\partial x_{1}^{\prime}}\right)+\frac{1}{8}\left(x_{1}^{\prime 2}+x_{2}^{\prime 2}\right)-\epsilon^{\prime} x_{1}^{\prime},
\end{aligned}
$$

where $\hbar_{\text {eff }}=\omega_{\mathrm{c}}^{1 / 3}$ is an effective Planck constant. If the effective Planck constant is small, the electron dynamics are expected to be described in terms of semiclassical or classical mechanics. By tuning the electric and magnetic fields, the effective Planck constant can be made arbitrarily small. However, due to the limitation of the experimental settings, attainable magnitude of the effective Planck constant may be limited. If the value of $\epsilon^{\prime}$ is chosen as before, the magnitude of the electric and magnetic fields can be written in terms of the effective Planck constant, such as $\bar{B}=2.35 \times 10^{5} \times \hbar_{\text {eff }}^{3} \mathrm{~T}$ and $\bar{E}=1.88 \times 10^{11} \times$ $\hbar_{\text {eff }}^{4} \mathrm{~V} / \mathrm{m}$, in the SI, and the switching occurs at the total energy $1.00 \times \hbar_{\text {eff }}^{2}$ a.u. above the Stark-saddle energy. The attainable magnitudes of the electric and magnetic fields highly depend on the experimental settings, but Raithel et al. have reported that, in their protocol, the attainable 
ranges of the electric and magnetic fields are $0.2-6.0 \mathrm{~T}$, and $10^{3} \sim 10^{5} \mathrm{~V} / \mathrm{m}$, respectively $[16,17]$. If our experiment is done under $\bar{B}=2.0 \mathrm{~T}$, and $\bar{E}=3.26 \times 10^{1} \mathrm{~V} / \mathrm{m}$, the effective Planck constant becomes $2.04 \times 10^{-2}$. Under the setting, the switching occurs at the total energy $4.17 \times 10^{-4}$ a.u. $\left(91.14 \mathrm{~cm}^{-1}\right)$ above the Stark-saddle energy. The energy at which the switching occurs corresponds to the principal quantum number $n \approx 71$ for the purely Coulombic problem without any external fields. Indeed, experimental studies $[16,17,23]$ have been carried out for investigating such highly excited states $(n \approx 44$ in Ref. [17] and $90 \leq n \leq 150$ in Ref. [23]) of ${ }^{85} \mathrm{Rb}$ in crossed electric and magnetic fields [17] and in an oscillating electric field [23]. However, angle-resolved measurements of the momentum distribution of escaping electrons pose a challenge even with state-of-the-art experimental techniques, and the predictions of the present work await their further development.

The authors thank Professor Masakazu Yamazaki for his valuable comments on this study. This work has been supported by JSPS, the Cooperative Research Program of "Network Joint Research Center for Materials and Devices," and a Grant-in-Aid for Young Scientists (B) (to T. H. No. 21740307), a Grant-in-Aid for Challenging Exploratory Research (to T. K. No. 25650044 and to M. T. No. 22654047 and No. 25610105), and a Grant-in-Aid for Scientific Research (B) (to T. K. No. 25287105 and No. 15KT0055) and (C) (to M. T. No. 24540394 and No. 26400421) from MEXT, the Office of the President of Hokkaido University, through a priority distribution fund for research support (to T. H.), a Nara Women's University Intramural Grant for Project Research (to M. T.).

*teramoto@es.hokudai.ac.jp

[1] R. Hernandez and W.H. Miller, Semiclassical transition state theory. A new perspective, Chem. Phys. Lett. 214, 129 (1993).

[2] S. Keshavamurthy and W. H. Miller, A semiclassical model to incorporate multidimensional tunneling in classical trajectory simulations using locally conserved actions, Chem. Phys. Lett. 205, 96 (1993).

[3] T. Komatsuzaki and M. Nagaoka, Study on "regularity" of the barrier recrossing motion, J. Chem. Phys. 105, 10838 (1996).

[4] T. Komatsuzaki and R. S. Berry, Dynamical hierarchy in transition states: Why and how does a system climb over the mountain?, Proc. Natl. Acad. Sci. U.S.A. 98, 7666 (2001).

[5] T. Uzer, C. Jaffé, J. Palacián, P. Yanguas, and S. Wiggins, The geometry of reaction dynamics, Nonlinearity 15, 957 (2002).

[6] M. W. Hirsch, C. C. Pugh, and M. Shub, Invariant Manifolds, Lecture Notes in Mathematics Vol. 583 (Springer-Verlag, Berlin, 1977).
[7] N. Fenichel, Persistence and smoothness of invariant manifolds for flows, Indiana University mathematics Journal 21, 193 (1971).

[8] N. De Leon, M. A. Mehta, and R. Q. Topper, Cylindrical manifolds in phase space as mediators of chemical reaction dynamics and kinetics. I. Theory, J. Chem. Phys. 94, 8310 (1991).

[9] N. De Leon, M. A. Mehta, and R. Q. Topper, Cylindrical manifolds in phase space as mediators of chemical reaction dynamics and kinetics. II. Numerical considerations and applications to models with two degrees of freedom, J. Chem. Phys. 94, 8329 (1991).

[10] N. De Leon, Cylindrical manifolds and reactive island kinetic theory in the time domain, J. Chem. Phys. 96, 285 (1992).

[11] N. De Leon and S. Ling, Simplification of the transition state concept in reactive island theory: Application to the HCN-CNH isomerization, J. Chem. Phys. 101, 4790 (1994).

[12] H. Waalkens, A. Burbanks, and S. Wiggins, Phase space conduits for reaction in multidimensional systems: $\mathrm{HCN}$ isomerization in three dimensions, J. Chem. Phys. 121, 6207 (2004).

[13] C. B. Li, A. Shojiguchi, M. Toda, and T. Komatsuzaki, Definability of No-Return Transition States in High Energy Regime above Threshold, Phys. Rev. Lett. 97, 028302 (2006).

[14] H. Teramoto, M. Toda, and T. Komatsuzaki, Dynamical Switching of a Reaction Coordinate to Carry the System through to a Different Product State at High Energies, Phys. Rev. Lett. 106, 054101 (2011).

[15] H. Teramoto, M. Toda, and T. Komatsuzaki, Breakdown mechanisms of normally hyperbolic invariant manifolds in terms of unstable periodic orbits and homoclinic/ heteroclinic orbits in Hamiltonian systems, Nonlinearity 28, 2677 (2015).

[16] G. Raithel and H. Walther, Ionization energy of rubidium Rydberg atoms in strong crossed electric and magnetic fields, Phys. Rev. A 49, 1646 (1994).

[17] G. Stania and H. Walther, Quantum Chaotic Scattering in Atomic Physics: Ericson Fluctuations in Photoionization, Phys. Rev. Lett. 95, 194101 (2005).

[18] J. Madronero and A. Buchleitner, Ericson Fluctuations in an Open Deterministic Quantum System: Theory Meets Experiment, Phys. Rev. Lett. 95, 263601 (2005).

[19] C. Simó, Effective computations in celestial mechanics and astrodynamics, CISM Courses Lect. 387, 55 (1998).

[20] V. I. Oseledec, A multiplicative ergodic theorem, Ljapunov characteristic numbers for dynamical systems, Trudy Mosk. Mat. Obsc. 19, 1971968.

[21] D. Ruelle, Ergodic theory of differentiable dynamical systems, Publ. Math. de l'I. H.É. S. 50, 27 (1979).

[22] J. Cresson and S. Wiggins, A $\lambda$-lemma for normally hyperbolic invariant manifolds, Regular Chaotic Dyn. 20, 94 (2015).

[23] Y. Kishimoto, M. Tada, K. Kominato, M. Shibata, S. Yamada, T. Haseyama, I. Ogawa, H. Funahashi, K. Yamamoto, and S. Matsuki, Systematic observation of tunneling field-ionization in highly excited Rb Rydberg atoms, Phys. Lett. A 303, 279 (2002). 